\title{
Formal equivalence between Tsallis and extended Boltzmann-Gibbs statistics
}

\author{
N. G. de Almeide* \\ Núcleo de Pesquisas em Física, Universidade Católica de Goiás, 74.605-220, Goiânia (GO), Brazil.
}

\begin{abstract}
A formal correspondence between the $q$-distribution obtained from the Tsallis entropy and non-maxwellian distributions obtained from the Boltzmann-Gibbs entropy is afforded.
\end{abstract}

PACS numbers: 05.70.Ce, 05.40.Fb, 13.85.Tp, 95.85.Ry

As is well known, generalized nonextensive statistics have increasingly received attention, and the physical needs for departure from Boltzmann-Gibbs statistical mechanics and thermodynamics have been investigated by several authors [1]. Since generalized nonextensive statistics are related to a new physics, it is a fundamental point to ask for the necessity of introducing nonextensivity at all. It is our purpose here to show a formal correspondence between the $\rho_{q}$ distribution associated with Tsallis entropy and the $\rho$ distribution obtained rightly from the Boltzmann-Gibbs entropy.

Tsallis entropy is defined by [2]

$$
S_{q}=k \operatorname{Tr} \frac{\rho-\rho^{q}}{q-1}
$$

where $\rho$ is the density matrix, $k$ is a positive constant and $q \in \mathbb{R}$ is the entropic index. Since the rule

$$
S_{q}(A+B)=S_{q}(A)+S_{q}(B)+(1-q) S_{q}(A) S_{q}(B) \geqslant 0
$$

is verified for two independent systems $A$ and $B$, the $q$ parameter has been interpreted as a measure of nonextensivity. When $q=1$ the Boltzmann-Gibbs entropy,

$$
S=-k \operatorname{Tr} \rho \ln \rho
$$

is recovered and corresponds to the extensive case. When $q<$ 1 and $q>1$ the system is said to possess superextensive and subextensive properties, respectively. The statistical operator $\rho_{q}$ obtained maximizing $S_{q}$ subject to the constraints

$$
\operatorname{Tr} \rho=1
$$

and

$$
\operatorname{Tr} \rho^{q} H=\langle E\rangle_{q}
$$

is

$$
\rho_{q}=\widehat{Z}_{q}^{-1}[1-(1-q) \beta H]^{\frac{1}{1-q}},
$$

where $H$ is the Hamiltonian, $\beta=1 / k T$ is the absolute temperature, $k$ is the Boltzmann constant and

$$
\widehat{Z}_{q}=\operatorname{Tr}[1-(1-q) \beta H]^{\frac{1}{1-q}}
$$

is the generalized partition function. The hat above $\widehat{Z}_{q}$ remind us of its operational character in contrast with the partition function $Z_{q}$. Once there is no ambiguity in the other operators appearing here, there is no need for using hat. Eq.(6) can be rewritten in the expanded form

$$
\rho_{q}=\widehat{Z}_{q}^{-1} \exp \left[-\sum_{n=1}^{\infty} \frac{(1-q)}{n}^{n-1}(\beta H)^{n}\right] .
$$

As usual, we want to maximize the Boltzmann-Gibbs entropy Eq. (3), subjected to certain constraints. If we know nothing about the system, except that $\operatorname{Tr} \rho=1$, then in the energy representation where $H|E\rangle=E|E\rangle$ all the states are equally likely, i.e., $P(E)=\langle E|\rho| E\rangle=$ constant. On the other hand, if we know the Hamiltonian $H$ for the system and the corresponding mean energy $\langle E\rangle=\operatorname{Tr} \rho H$, thus using Lagrange multipliers it is straightforward to show that the entropic form Eq.(3) leads to the Maxwell-Boltzmann distribution $P(E)=Z^{-1} \exp (-\beta E)$. If additional measurements are made on the system such that, besides the mean energy, we also know the mean square, or more generally, the central moments of order $p$, i.e., $\left\langle(\Delta E)^{p}\right\rangle=\left\langle(H-\langle E\rangle)^{p}\right\rangle$, we can impose this knowledge as additional constraints. Note that, classically, if we know all order of moments, we can precisely characterize the distribution function. Thus, let us consider $\left\langle(\Delta E)^{n}\right\rangle, n$ integer, as new constraints and redefine the energy level such that now we have the moments

$$
\left\langle(\Delta E)^{n}\right\rangle=\operatorname{Tr} \rho H^{n} .
$$

When we vary $\rho$ in Eq. (3) and in those for the constraints Eq.(9), multiplying each constraint by the undetermined Lagrange multipliers $\beta_{0}, \beta_{1}, \beta_{2}, \ldots \beta_{n}$ and adding the result, we obtain

$$
\operatorname{Tr}\left(1+\sum_{n=0}^{\infty} \beta_{n} H^{n}+\ln \rho\right) \delta \rho=0 .
$$

Since all variations are independent and $\delta \rho$ is arbitrary, it follows the extended (non-maxwellian) distribution

$$
\ln \rho=-1-\sum_{n=0}^{\infty} \beta_{n} H^{n}
$$

or, equivalently

$$
\rho=\widehat{Z}^{-1} \exp \left(-\sum_{n=1}^{\infty} \beta_{n} H^{n}\right),
$$

where

$$
\widehat{Z}=\operatorname{Tr} \exp \left(-\sum_{n=1}^{\infty} \beta_{n} H^{n}\right)
$$


is the partition function. In the energy representation, Eq.(12) and Eq.(13) reads, respectively,

$$
P(E)=Z^{-1} \exp \left(-\sum_{n=1}^{\infty} \beta_{n} E^{n}\right)
$$

and

$$
Z=\sum_{E} \exp \left(-\sum_{n=1}^{\infty} \beta_{n} E^{n}\right) .
$$

Eq.(15) can be used to obtain all the Lagrange multipliers considering formally $E^{k}=Y_{k}$ as independent variables:

$$
\beta_{k}=-\frac{\partial \ln Z}{\partial E^{k}} .
$$

The formal correspondence between the $q$-distribution Eq.(8) and the extended distribution Eq.(12) can be found, both leading to the same result, imposing

$$
\beta_{n}=\frac{(1-q)^{n-1}}{n} \beta^{n} .
$$

From Eq. 117) we see that, in general, in order to get $\rho=\rho_{q}$, an infinite number of constraints is needed. However, if the energy dispersion is not significative, being the distribution highly concentrated around the mean energy, or, equivalently, if all the Lagrange multipliers except $\beta_{1}$ are very small, the sum in Eq.(12) and Eq.(8) can be truncated, and $q$ in Eq. (17) will differ only slightly from the unity. Actually, three decades ago, to solve discrepancies between the observed and predicted fluxes of solar neutrino, D.D. Clayton [3] proposed that the Boltzmann factor $\exp (-\beta E)$ was modified to $\exp \left(-\beta E-\beta_{2} E^{2}\right)$, which can be rewritten as

$$
P(E)=Z^{-1} \exp \left[-\beta E-\delta(\beta E)^{2}\right],
$$

where $\delta$ is a small parameter needed to fit experimental results. As Clayton noted, $\delta \sim 0.01$ is enough to explain the solar neutrino fluxes. The agreement between the extended Boltzmann-Gibbs statistics and the result by Clayton can be obtained truncating Eq.(14) after the quadratic term:

$$
P(E)=Z^{-1} \exp \left(-\beta_{1} E-\beta_{2} E^{2}\right),
$$

with

$$
Z=\sum_{E} \exp \left(-\beta_{1} E-\beta_{2} E^{2}\right)
$$

In the same way, the result by Clayton follows from Tsallis statistics choosing $q=1-2 \delta$. In this context, since $q<1$ the system is regarded as superextensive, and the inadequacy of Boltzmann-Gibbs entropy is claimed [4]. Also, a possible correspondence between the Clayton parameter and the anomalous diffusion phenomena related to the nonextensive entropy Eq. (11) was discussed in Ref.[5]. Further discussion following this approach can be found in [6], all of them reinforcing the nonextensivity. However, we see that Clayton proposals can be derived from the Boltzmann-Gibbs entropy simply considering the mean square energy as an additional constraint. The formal correspondence between Tsallis and the extended Boltzmann-Gibbs statistics, in this case, is obtained from Eq. (17): $\beta_{1}=\beta$ and $\beta_{2}=\frac{(1-q)}{2} \beta^{2}$. Note that in our derivation the Clayton parameter acquires a natural meaning, stemming from the energy fluctuations of the system, which we consider as a new constraint in the maximization of the Boltzmann-Gibbs entropy. Besides, as a general rule, the factor correcting Boltzmann-Gibbs statistics is negligible whenever the dispersion be highly concentrated around the mean energy, i.e., $(\Delta E)^{2} \cong 0$, which is undoubtedly the most common situation. On the other hand, corrections to the Boltzmann factor are expected whenever the fluctuations become dominant. Also note that to recover the Boltzmann factor from the extended Boltzmann-Gibbs entropy, it is necessary to impose that all moments $(\Delta E)^{n}$ become successively small, implying that the energy does not deviate very much from the mean value. This is specially evident if we rewrite Eq.(14) as

$$
P(E)=Z^{-1} \exp \left(-\sum_{n=1}^{\infty} \widetilde{\beta}_{n}(E-\bar{E})^{n}\right)
$$

where $\widetilde{\beta}_{n}$ are the new Lagrange multipliers that maximize the Boltzmann-Gibbs entropy subject to the constraints $\left\langle(\Delta E)^{n}\right\rangle=\left\langle(H-\bar{E})^{n}\right\rangle$. To relate the constraints in Eq. (21) with those in Eq. (14) we have to solve the following equality

$$
\left.\left.\sum_{n=1}^{\infty} \widetilde{\beta}_{n}(E-\bar{E})^{n}\right)=\sum_{n=1}^{\infty} \sum_{k=1}^{n} \frac{n !}{(n-k) ! k !} \widetilde{\beta}_{n} E^{k}(-\bar{E})^{n-k}\right),
$$

and, for the special case of the Clayton proposal discussed above, Eq.(21) reduces to

$$
P(E)=Z^{-1} \exp \left[-\widetilde{\beta}_{1}(E-\bar{E})-\widetilde{\beta}_{2}(E-\bar{E})^{2}\right],
$$

which, when compared to Eq. (18), gives the new constraints: $\widetilde{\beta}_{2}=\delta \beta^{2}$ and $\widetilde{\beta}_{1}=\beta+2 \delta \beta^{2} \bar{E}$. On the other hand, Eq.(6) and its corresponding distribution function in the energy representation, is obtained from Tsallis entropy with the constraint given solely by the mean energy, and the Boltzmann factor is recovered from Tsallis entropy simply establishing the limit $q \rightarrow 1$ without reference to the variance or the moments $(\Delta E)^{n}$. Also, note that, for all systems having the same absolute temperature, the Lagrange multiplier associated with the mean energy is the same, that is $\beta_{1}=\beta$, while, in general, the others Lagrange multipliers $\beta_{n+1}$ will be different for each system. This is true for the generalized distribution stemming from Boltzmann-Gibbs entropy as well as for the generalized distribution stemming from Tsallis entropy, thus giving rise to free parameter(s). That this must be, in general, true is particularly illuminating from the approach presented here. In fact, based on Eq.21) we can immediately see that, since the 
second central moment is related to the mean square deviation, two or more distributions having the same temperature, i.e., the same mean energy, can be distinguished by the energy dispersion. However, if the mean energy and its dispersion is not enough to distinguish between two or more distributions, the third central moment, being proportional to the coefficient of skewness, which is a measure of the asymmetry of the probability distribution, can be used. If even the third central moment is not enough, we can use the fourth central moment, which is proportional to the kurtosis, and so on. Therefore, we can conclude that by maximizing the Boltzmann-Gibbs entropy we will find, in general, that the values for the Lagrange multipliers in Eq. (16), related to energy fluctuations, will depend on the system under consideration, turning difficult, if not impossible, to assert a priori their values. This same difficulty applies to the $q$ entropic index, which is argued to be related to the microscopic dynamics intrinsic to each system.

In summary, we have shown a formal correspondence between Tsallis and the extended Boltzmann-Gibbs statistics. This formal correspondence is obtained assuming that the infinite constraints maximizing Boltzmann-Gibbs entropy, and leading to an extended distribution function, are related to the $q$ entropic index appearing in Tsallis entropy in such a way that both the distribution function obtained by maximizing Tsallis entropy and the distribution function obtained by maximizing Boltzmann-Gibbs entropy become identical. Also, we have suggested a relationship between the $q$ entropic index and the higher order moments of the extended Boltzmann-
Gibbs entropy. As a final remark, we stress that the formal equivalence between Tsallis and the extended BoltzmannGibbs statistics demonstrated here rises an interesting question related to a possible pseudo nonextensive situation, a subject hereafter deserving more attention.

I would like to thank VPG/UCG and CNPq for partial support.

* Electronic address: norton@ @esquisador.cnpq.br

[1] See, for example, Europhysics News 36, 183 (2005), Special Issue on Nonexttensive Statistical Mechanics: New trends, new perspectives, eds. J.P. Boon and C. Tsallis; Nonextensive entropy - Interdisciplinary applications, eds. M. Gell-Mann and C. Tsallis, Oxford University Press, New York, 2004; Braz. Journal of Physics, Vol. 29, n.1, March 1999: Special Issue on Nonexttensive Statistical Mechanics and Thermodynamics, eds. S. R. A. Salinas and C. Tsallis.

[2] C. Tsallis, J. Stat. Phys. 52, 479 (1988).

[3] D. D. Clayton, Nature 249, 131 (1974).

[4] G. Kaniadakis, A. Lavagno, and P. Quarati, Physics lett. B 369, 308 (1996).

[5] G. Kaniadakis, A. Lavagno, M. Lissia, and P. Quarati, Physica A 261, 359 (1998).

[6] P. Quarati, A. Carbone, G. Gervino, G. Kaniadakis, A. Lavagno, and E. Miraldi, Nucl. Phys. A 621, 345c (1997); M. Coraddu, G. Kaniadakis, A. Lavagno, M. Lissia, G. Mezzorani, and P. Quarati, Braz. J. Phys. 29, 153 (1999). 